\title{
"SAMs meet MEMS": surface modification with self-assembled monolayers for the dry-demolding of photoplastic MEMS/NEMS
}

\author{
B.J. Kim ${ }^{1, *}$, G.M. Kim ${ }^{1}$, M. Liebau ${ }^{2}$ J. Huskens ${ }^{2}$, D.N. Reinhoudt ${ }^{2}$ and J. Brugger ${ }^{1}$ \\ Strategic Research Orientation Nanolink ${ }^{1} \&$ Supramolecular Chemistry and Technology Group ${ }^{2}$, \\ MESA+ Research Institute, University of Twente \\ P.O.Box 217, 7500AE, Enschede, The Netherlands \\ Phone: +31-53-489-5652, Fax: 2575, e-mail: j.p.brugger@el.utwente.nl
}

\section{INTRODUCTION}

The fabrication of micro-electro-mechanical-systems (MEMS) by means of surface microfabrication techniques often involves a final etch step to release freestanding and movable parts, or to detach microstructures completely from the surface. Selective removal of a sacrificial layer underneath the structural layer by etching is the most commonly used method, although it encompasses several manufacturing problems. One problem when using wet chemistry for example is the risk of permanent attachment of slender structures to the underlying substrate after drying, other problems are the etch selectivity between the sacrificial and structural layers and risk of surface contamination, in particular when chemically active surfaces for (bio)chemical sensors are involved.

Dry-etchable organic layers have therefore been introduced in MEMS fabrication techniques to function as sacrificial layer in order to prevent release-related stiction in free-standing or movable micromechanical elements, and also to prevent surface contamination [1],[2]. In another application the grafting of densely packed organic molecules on microstructure surfaces is used to obtain nanometer-thick monolayers which enhance the tribological performance of movable MEMS parts [3]

In this contribution we demonstrate the use of selfassembled monolayers (SAMs) as anti-adhesion coating to assist the removal of photoplastic MEMS/NEMS with a patterned metal layer from the surface without wet chemical sacrificial layer etching [4], so-called 'dry-demolding'. The SAMs functionality here is to reduce the stiction between the surface and a thin evaporated metal film. The double-layer $\mathrm{SAM} /$ metal provides enough stability to support subsequent micromachining steps.

Our approach differs from other known methods because the sacrificial organic layer is only $\sim 1.5 \mathrm{~nm}$ thick which is a condition to be able to cover surface topographies down to the nanometer scale at highest possible conformality. We will show that this enables e.g. the precise replication and demolding of functionalized nanoscale structures as required for scanning probe instruments, in particular for the scanning near-field optical microscope (SNOM). Furthermore, the described method allows for the fabrication of a variety of novel surface micromachined structures, including highaspect ratio plastic devices with embedded bottom-side metal micro- and nanoscale electrode pattern.

An additional and particularly interesting method is to use the dry-release technique on microcontact printed $(\mu \mathrm{CP})$ SAM pattern. This allows to transfer a spatial variations of surface adhesion properties (as defined by the SAM pattern) directly into a metal structure by simple mechanical thin-film rupture without the need for additional process steps.

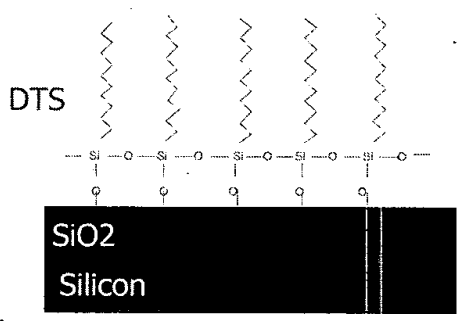

Figure 1: Schematic representation of a self-assembled monolayer (SAM) of dodecyltrichlorosilane (DTS) on a hydroxylated surface.

\section{FORMATION OF SAM AND ALUMINUM/SU-8 PROCESS}

\section{SAM}

Organosilicon derivatives $\mathrm{RnSiX3}-\mathrm{n}$, where $\mathrm{X}$ is chloride or alkoxy, are well known to form SAMs on hydroxylated surfaces [5]. In our case, we start with a (1.00) single crystal silicon wafer that is covered by a 300 -nm-thick thermally grown silicon dioxide $\mathrm{SiO} 2$ layer.

Then, a $\sim 1.5$-nm-thick SAM is formed on the $\mathrm{SiO} 2$ surface by immersing it into a solution of typically $5-10 \mathrm{x}$ $10^{-3} \mathrm{M}$ dodecyltrichlorosilane (DTS) in dry toluene for about 2 hours. Thereby, a network of molecules is formed connecting them to the surface and to each other by covalent bonds as shown in Figure 1. The main observation is a drastic reduction of the surface wettability as revealed by contact angle measurement. SAMs of alkytrichlorosilanes are stable against detergents and organic solvents and they withstand also acidic solutions, whereas under basic conditions the monolayers can suffer deterioration. Advancing contact angles of $112^{\circ}$ indicated the hydrophobic nature of the prepared layers. A low hysteresis of $17^{\circ}$ between the advancing and receding contact angles $\left(95^{\circ}\right)$ showed dense packing of SAMs. For comparison, SAMs of 3-aminopropyltriethoxysilane (APTES) were also prepared. APTES is forming more hydrophilic layers having an advancing contact angle of $67^{\circ}$. Thus, the application of different types of monolayers enables the tuning of the surface properties, such as wettability and

* present address: Institute of Industrial Science, University of Tokyo. CIRMM (Center for International Research on MicroMechatronics) 4-6-1 Komaba, Meguro-ku, Tokyo 153-8505, Japan 
adhesion, of silicon wafers. The present study applies SAMs of DTS as non-adhesive layers to reduce the adhesion between metal and wafer.

\section{ALUMINUM PATTERN AND SU-8 PROCESS}

After the SAM has been formed, a 100 -nm-thick aluminum ( $\mathrm{Al}$ ) film is evaporated and micropatterned by lithography and wet-etching. As an alternative patterning method we used evaporation through shadowmasks which formed sub-micron sized dots on large areas directly onto the ultrathin SAM layer. The advantage of this method is that no pattern transfer step is necessary, which makes this resistless method very fast and intrinsically clean and contaminationfree [6].

The actual MEMS/NEMS microstructure is then made by a epoxy-type photoresist containing the resin Epon-SU-8 which is very suitable for the production of high-aspect ratio micromechanical structures formed from ultrathick films using standard UV-lithography [7]. In our case, the SU-8 process steps are done directly on the SAM/Al sandwich layer. A schematic illustration of the resulting sandwich layer consisting of $\mathrm{SiO} 2 / \mathrm{SAM} / \mathrm{Al} / \mathrm{SU}-8$ is shown in Figure 2. The role of the SAM as molecular 'nanocarpet' in this configuration is to reduce the adhesion between $\mathrm{Al}$ and $\mathrm{SiO} 2$, but to provide enough stability to support all subsequent process steps, such as multiple resist spinning, thermal steps, development and etching.
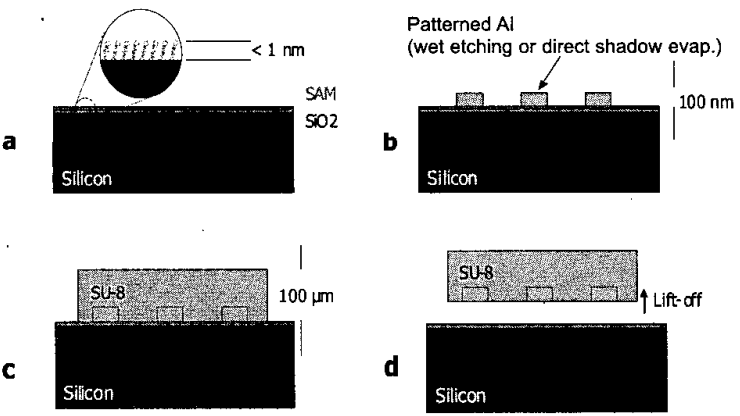

Figure 2: Schematic representation of the sandwich layer formation and dry-release process.

An oxidized silicon wafer is subsequently covered by a SAM (a), 100-nm thick Al film (patterned or not) (b) and $\sim 100-\mu m$ thick SU-8 structure (c). low adhesion forces between SAM and Al/SU-8 allows to lift the complete structure off the surface with the metal pattern embedded in the $S U-8(d)$.

After post-exposure bake the micro-structured SU-8 structures are removed from the surface by using microtweezers. We have designed our system so that the SU-8 structure can be easily lifted off the surface together with the $\mathrm{Al}$ pattern. A unique asset of this technique is that the $\mathrm{Al}$ film is embedded in the SU-8 and that the metal surface is very smooth with less than $0.8 \mathrm{~nm}$ mean surface roughness as measured by atomic force microscopy (AFM). An AFM image and a line profile over a typical $100-\mathrm{nm}$ thick and $5 \mathrm{um}$ sized lithographically defined pattern are shown in Figure 3.

Instead of lithographical patterning of $\mathrm{Al}$ using chemical etching, we patterned sub-micron $\mathrm{Al}$ dots arrays on the SAMed surface using resistless direct shadow mask technique. Figure 4 (a) shows a sub-micron metal pattern that has been directly deposited on the organic monolayer by a shadow mask technique. Lift-off and embedded $\mathrm{Al}$ dots in SU-8 are also shown in Figure 4 (b).

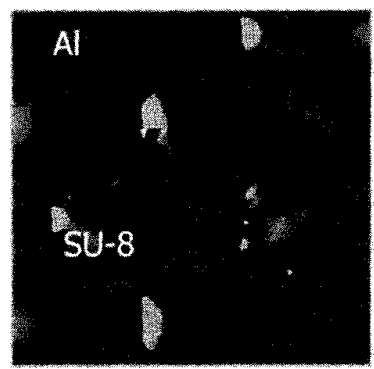

Figure 3: Atomic force microscope (AFM) image showing a 15- $\mu \mathrm{m} \times 15-\mu \mathrm{m}$ section of the SU-8 microstructure released from the SiO2/SAM surface with the Al film. The Al film here has been previously structured lithographically.
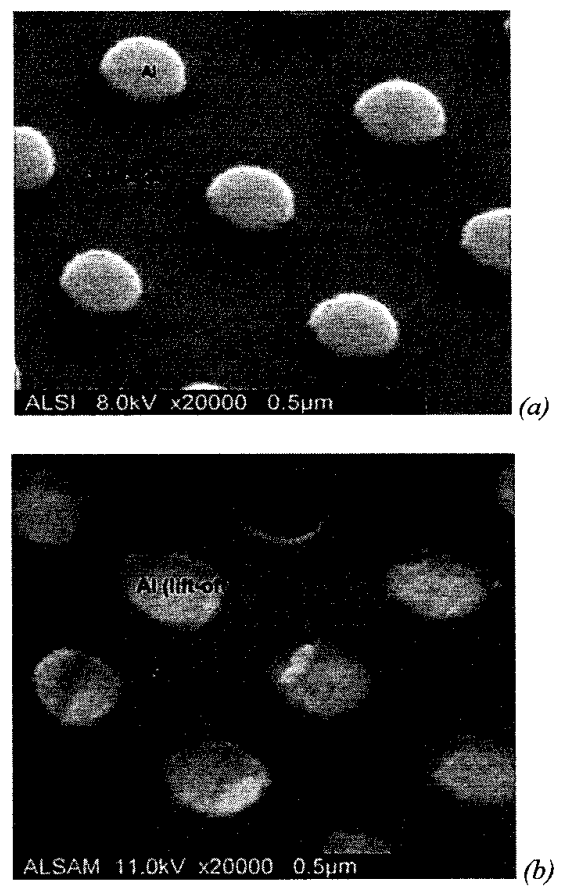

Figure 4: SEM images of (a) Shadow mask evaporated Al dots arrays on SAM surface (b) lift-off and embedded dots in SU-8.

\section{PATTERNED SAM AND AL/SU-8 PROCESS}

An interesting additional method is to use a patterned monolayer defined by microcontact printing $(\mu \mathrm{CP})$ instead of a self-assembled homogenous monolayer covering the entire surface. Here, the surface adhesion properties vary between strong (without SAM) and weak (with SAM) according to the printed pattern. This enables to transfer a structured SAM pattern directly into a corresponding metal structure without intermediate transfer steps. The schematic view of this process is shown in Figure 5.

A patterned SAM is formed on a silicon oxide surface by contact printing and is covered by a thin evaporated metal 
film (Al, $20 \mathrm{~nm}$ in thickness). A thick epoxy is then spun and structured by photolithography and peeled off the surface. Because of reduced local adhesion between SAM and silicon oxide, the removal step peels off the micro-structured epoxy with the metal film attached to the epoxy at the locations where the SAM has been previously defined. This results in a complementary metal pattern on the surfaces, which can be used directly or could form a robust mask for subsequent postprocessing. Further work to reduce dimensions has to be which includes detailed knowledge on the rupture of thin filrns.

A

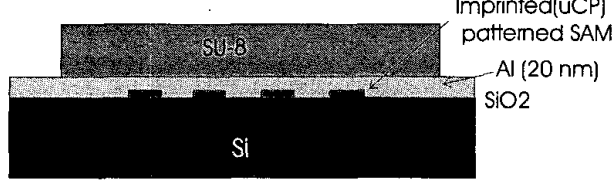

B

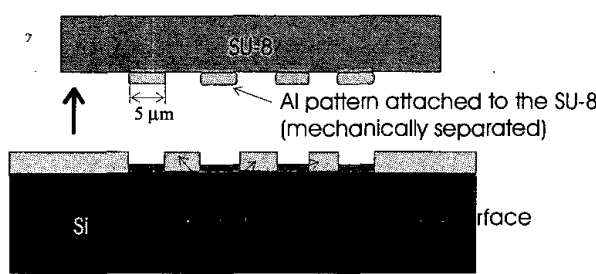

Figure 5: Schematic illustration of (a) microcontact printing $(\mu C P)$ to define a sandwich layer of patterned $S A M$, $A l$ and $S U-8$, and (b)method of layer separation induced by different surface adhesion properties.

As shown in Figure 6,5-micron wide and 20-nm-thin aluminum lines are still attached to the silicon dioxide surface forming the complementary pattern due to local adhesion differences.

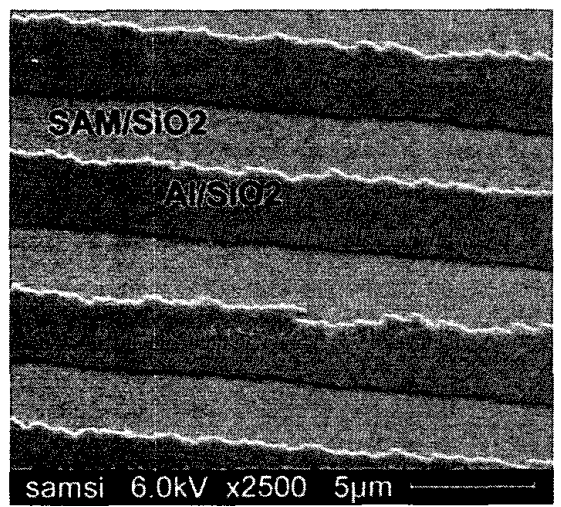

Figure 6: SEM micrograph of an Al pattern which has been fabricated by mechanical lift-off from a patterned SAM layer (microcontact printed - $\mu C P$ ) without additional transfer steps.

\section{DEMOLDING OF METALIZED NANOPROBE FOR SNOM}

The smooth and nanometer precise demolding of metallized SU-8 structures has also been used to improve the integrated microfabrication of nanoprobes for near-field optical microscopy. The novel SNOM probe is composed of a light-guiding pyramidal tip (SU-8) covered by a lightabsorbing layer $(\mathrm{Al})$ with a postprocessed nanoscale aperture at the tip apex (by focused ion beam). This process is adapted from [8] for low-cost photoplastic atomic force microscope (AFM) probes and extended here for the demolding of the SU-8 nanoprobe jointly with the metal layer, which would allow the low-cost fabrication of SNOM probes with high brightness [9]. A typical microfabricated probe is shown in Figure 7.

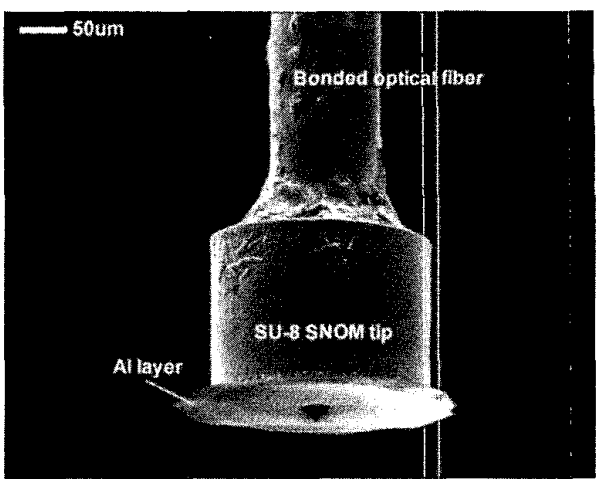

Figure 7: SEM micrograph of a microfabricated photoplastic SNOM probe attached at the end of an optical fiber. The Al layer forms an integral part of the probe and has been made using the combined SAM/MEMS process.

\section{CONCLUSIONS}

Ultrathin organic self-assembled monolayers were used to functionalize surfaces prior to replication and de-molding processes. This technique is useful for many MEMS related applications, SPM probe fabrication using nanomolding, double side patterned MEMS structures, unconventional patterning of polymer devices, contamination-free electrode formation, etc.

SAMs are used as a releasing agent without sacrificial layer etching for surface micro-machined MEMS structures allowing to replicate structures down to the nanometer scale. This technique enables furthermore to directly transfer a $\mu \mathrm{CP}$ SAM pattern into a metal structure.

\section{REFERENCES}

[1] U. Srinivasan, M. R. Houston, R. T. Howe, R. Maboudian, "Alkyltrichlorosilane-based self-assembled monolayer films for stiction reduction in silicon micromachines", $J$. Microelectromechanical Systems, Vol.2, 2 (1998), 252-260

[2] H.-J. Suh, P. Bharathi, D.J. Beebe, J.S. Moore, "Dendritic material as a dry-release sacrifical layer", Journal of Microelectromechanical Systems, Vol. 9, No. 2, June 2000

[3] Z. Rymuza, "Control tribological and mechanical properties of MEMS surface. Part 1: critical review", Microsystem Technologies, 5 (1999), 173-180

[4] B.J. Kim, M. Liebau, J. Huskens, D.N. Reinhoudt and J. Brugger, Proceedings MNE 2000, Jena, Germany, submitted to Microelectronic Engineering

[5] J. Sagiv et al. J. Am. Chem. Soc. (1980), 102, 92 
[6] J. Brugger, W. Berenschot, S. Kuiper, W. Nijdam, B. Otter, M. Elwenspoek, "Resistless patterning of sub-micron structures by evaporation through nanostencils", Microelectronic Engineering, 53 (2000) 403-405

[7] K.Y.Lee et al. J.Vac.Sci.Technol. B, 13(6), Nov/Dec 1995; M. Despont et al., MEMS97 workshop, and H. Lorentz et al., Sensors and Actuators A, 64, 33, 1998,

[8] G. Genolet, J. Brugger, M. Despont, U. Drechsler, P. Vettiger, N. F. de rooij, and D. Anselmetti, "Soft, entirely photoplastic probes for scanning force microscopy", Rev. Sci. Instr. Vol. 70, No. 5, (1999) 2398-2401

[9] B. Kim, J.W. Flamma, E.S. ten Have, M.F. Garcia-Parajo, N.F. van Hulst, J. Brugger, "Molded photoplastic probes for Near-field optical applications", Journal of Microscopy (in press). 